\title{
Plexiform ameloblastoma of mandible - case report
}

\author{
Ajinkya Varkhede ${ }^{1}$, JV Tupkari ${ }^{2}$, MS Mandale ${ }^{3}$, Manisha Sardar ${ }^{4}$ \\ ${ }^{1}$ P.G. student. Government Dental College \& Hospital, Mumbai, Maharashtra, India. \\ ${ }^{2}$ Prof.\& H.O.D., Government Dental College \& Hospital, Mumbai, Maharashtra, India. \\ 3 Asso. Prof, Government Dental College \& Hospital, Mumbai, Maharashtra, India. \\ 4. Senior Lecturer, Government Dental College \& Hospital, Mumbai, Maharashtra, India.
}

Correspondence:

Dr. Ajinkya S Varkhede, PG student

Department of Oral Pathology and Microbiology

Room no 301, Government Dental College and Hospital,

Mumbai 400001 Maharashtra

Email address-varkhedeajinkya260@gmail.com

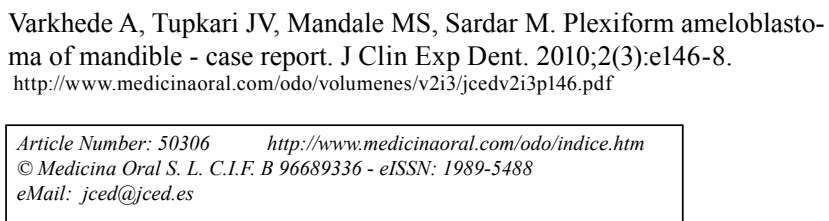

\begin{abstract}
Ameloblastoma, a benign epithelial odontogenic tumor, is locally aggressive. This tumor comprises about $1 \%$ of tumors and cysts arising in the jaws. It appears most commonly in the third to fifth decades and with equal frequency between sexes. Ameloblastoma prevalently occurs in the mandibular molar and the ramus areas. Recurrence frequently appears after inadequate treatment. They are usually benign in growth pattern but frequently invade locally and occasionally metastasize. In the present study, a case of unusually large plexiform ameloblastoma was presented with its clinical, radiological, histological features and treatment modalities, and this is the addition of one more case in the literature.
\end{abstract}

Key words: Ameloblastoma, mandible, odontogenic tumors. 


\section{Introduction}

Ameloblastoma is a true neoplasm of odontogenic epithelium (1). It represents about $1 \%$ of all oral ectodermal tumors and $9 \%$ of odontogenic tumors (2). It is an aggressive neoplasm that arises from remnants of the dental lamina and dental organ (odontogenic epithelium) (3). Most ameloblastomas develop in the molar-ramus region of the mandible with $70 \%$ of these arising in the molar-ramus area and they are occasionally associated with unerupted third molar teeth (4). Ameloblastoma appears most commonly in the third to fifth decades but the lesion can be found in any age group including children $(2,5)$.

The chief histopathological variants of ameloblastoma are the follicular and plexiform types, followed by the acanthomatous and granular cell types. Uncommon variants include desmoplastic, basal cell, clear cell ameloblastoma, keratoameloblastoma and papilliferous ameloblastoma (5). It is well known that ameloblastoma can be radiologically unilocular or multilocular radiolucency with a honeycomb or soap bubble appearance.

The plexiform pattern is less aggressive and has a significantly lower recurrence rate (6).

\section{Case report}

A 12 year old girl had been reported to the Department of Oral Pathology and Microbiology with complaint of swelling on left side of face since last 2 years. The medical history was unremarkable.

Clinical examination revealed diffuse, smooth - surfaced, hard swelling on left side of face. It extends from the zygomatic region to the inferior border of mandible superoinferiorly, and from the corner of mouth to the angle of mandible anteroposteriorly. It was large, expansive, and painless. It was covered by normal mucosa (Fig.1).
Intraorally, the swelling extends from distal of first molar posteriorly. Swelling results in obliteration of buccal vestibule.

Panoramic radiography showed a large multilocular radiolucent area occupying the left mandible from the first molar tooth to the neck of condylar process and the coronoid process including the left ascending ramus area. Root resorption was observed in the first molar. The base of the mandible and the anterior border of the ramus was damaged and thinned (Fig.2).

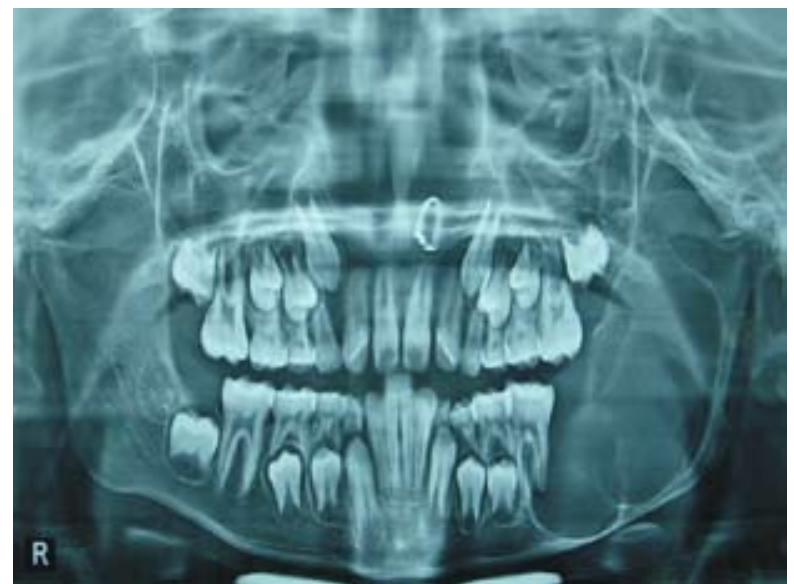

Fig. 2. Panoramic radiogram revealed a large multilocular radiolucent area extending from the left first molar to the left coronoid process including the left ascending ramus area. Permanent maxillary and mandibular canines and premolars are seen below the deciduous teeth.

The histopathological processing of the tumor revealed a plexiform ameloblastoma predominantly composed of epithelium arranged as a tangled network of anastomosing strands. The cords or sheets of epithelium are bounded by columnar or cuboidal ameloblast like cells surrounding more loosely arranged epithelial cells. The
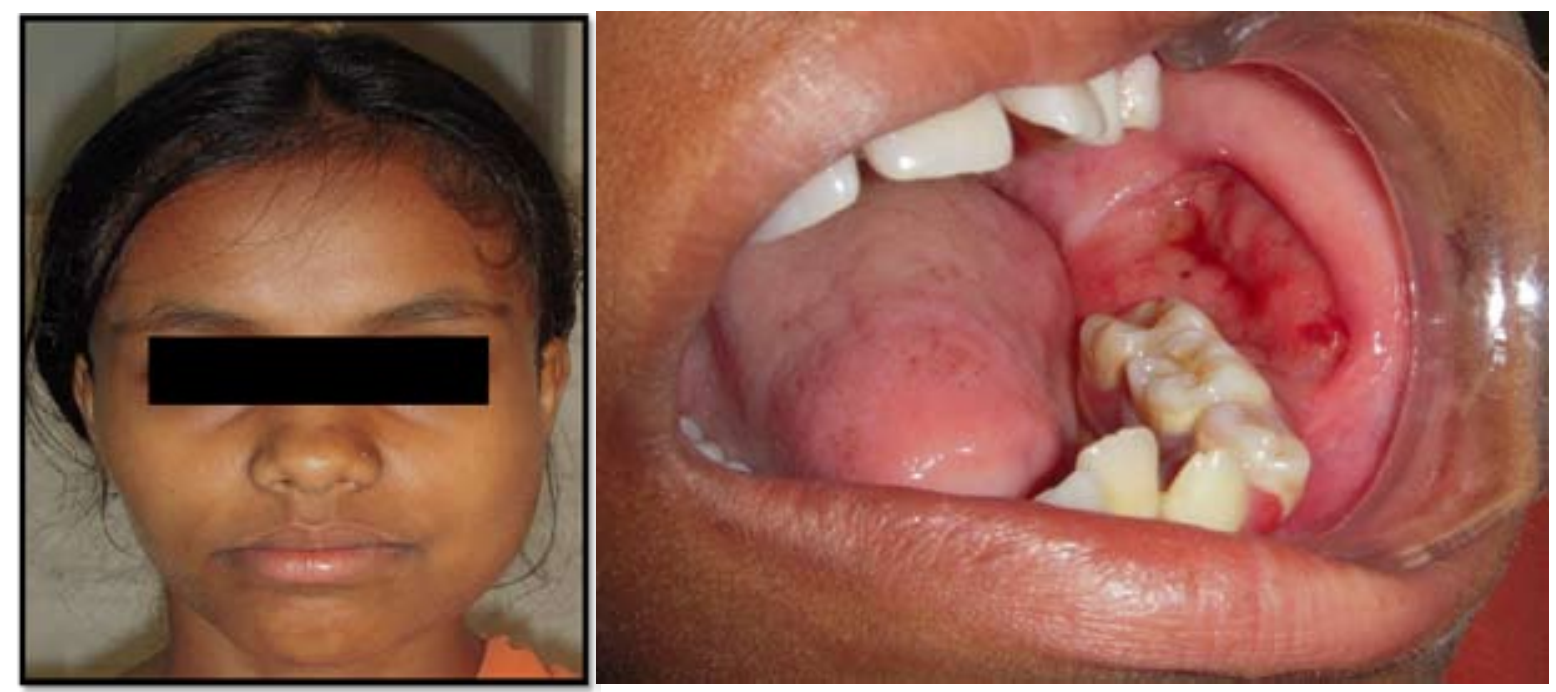

Fig.1. A)Etra oral view shows swelling on left side of face; B) Intraoral view shows obliteration of left buccal vestibule 


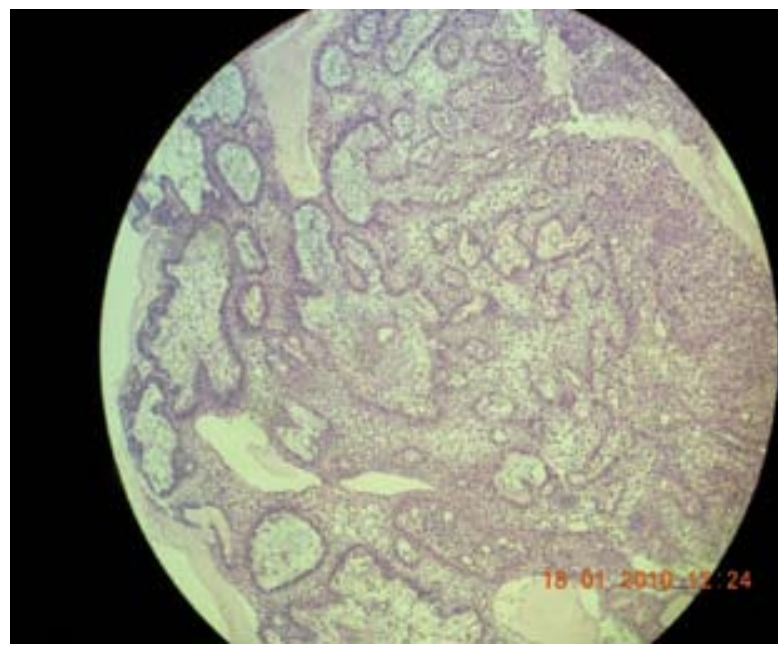

Fig.3. Anastomising cords of odontogenic epithelium

supporting stroma is loosely arranged and vascular (Fig.3).

Under general anesthesia and nasoendotracheal intubation and all aseptic precautions, tumor mass was exposed buccally and lingually. After extraction of lower first premolar, osteotomy cut was placed and completed buccally and lingually. Thus tumor mass was removed along with bone margin of $1.5 \mathrm{~cm}$. Microvascular free fibula graft was harvested from right leg along with peroneal artery and vessels. Graft dimension was $14 \mathrm{~cm} X 3.5 \mathrm{~cm} X 3 \mathrm{~cm}$. Hemostasis was achieved, vacuum drain was secured and closure was done in layers. Antibiotics, analgesics and anti-inflammatory drugs were given postoperatively. Both wounds healed unevenly and sutures were removed on 7 th postoperative day. Patient has been kept under periodic follow up since then. No recurrence had been reported till date.

\section{Discussion}

Ameloblastoma is a benign epithelial odontogenic tumor but is often aggressive and destructive, with the capacity to attain great size, erode bone and invade adjacent structures (7). Although the term ameloblastoma was coined by Churchill in 1933, the first detailed description of this lesion was by Falkson in 1879 (7). It is the most common odontogenic tumor although it represents only about $1 \%$ of tumors and cysts of the jaws (5).

In the mandible ( $80 \%$ of ameloblastomas), $70 \%$ are located in the area of the molars or the ascending ramus, $20 \%$ in the premolar region, and $10 \%$ in the anterior region (2). About $10-15 \%$ of ameloblastomas are associated with a non-erupted tooth. In the present case, a large plexiform ameloblastoma found in the ascending ramus and molar region of the mandible and it was not associated with a non-erupted tooth.

Ameloblastoma appears equal frequency between sexes, although a higher frequency in females than in males has been described (7). In our case, the patient was female and was in second decade of her life.

Clinically, it frequently manifests as a painless swelling, which can be accompanied by facial deformity, malocclusion, ulceration and periodontal disease and paresthesia of the affected area. In our case, clinical examination revealed a large, expansive mass in the ascending ramus and molar region of the mandible. The swelling was hard, painless to palpation and covered by normal mucosa.

Histologically, ameloblastoma is characterized by the proliferation of epithelial cells arranged in a collagenous fibrous connective tissue stroma of conjunctive vascular tissue in locally invading structures that resemble the enamel organ at different stages of differentiation (7). The tumor found in our patient was an ameloblastoma of the plexiform type. The term "plexiform" refers to the appearance of anastomosing islands of odontogenic epithelium in contrast to a follicular pattern.

\section{References}

1. Torres-Lagares D, Infante-Cossío P, Hernández-Guisado JM, Gutiérrez-Pérez JL. Mandibular ameloblastoma. A review of the literature and presentation of six cases.

Med Oral Patol Oral Cir Bucal. 2005; 10:231-8.

2. Adebiyi KE, Ugboko VI, Omoniyi-Esan GO, Ndukwe KC, Oginni FO. Clinicopathological analysis of histological variants of ameloblastoma in a suburban Nigerian population. Head Face Med. 2006; 2:42. 3. Tozaki M, Hayashi K, Fukuda K. Dynamic multislice helical CT of maxillomandibular lesions: distinction of ameloblastomas from other cystic lesions. Radiat Med. 2001; 19:225-30.

4. Ogunsalu C, Daisley H, Henry K, Bedayse S, White K, Jagdeo B, et al. A new radiological classification for ameloblastoma based on analysis of 19 cases. West Indian Med J. 2006; 55:434-9.

5. Nakamura N, Mitsuyasu T, Higuchi Y, Sandra F, Ohishi M. Growth characteristics of ameloblastoma involving the inferior alveolar nerve: a clinical and histopathologic study. Oral Surg Oral Med Oral Pathol Oral Radiol Endod. 2001;91:557-62.

6. Gümgüm $\mathrm{S}, \mathrm{Ho}^{\circ}$ gören $\mathrm{B}$. Clinical and radiologic behaviour of ameloblastoma in 4 cases. J Can Dent Assoc. 2005;71:481-4.

7. Iordanidis S, Makos C, Dimitrakopoulos J, Kariki H. Ameloblastoma of the maxilla. Case report. Aust Dent J. 1999;44:51-5. 